\title{
TIMING PERFORMANCE OF A VACUUM PHOTOTRIODE
}

DAWN E. LESLIE, IGNACIO YASELLI AND PETER R. HOBSON*

School of Engineering and Design, Brunel University, Uxbridge UB8 3PH, UK

The timing performance of a vacuum phototriode (VPT) has recently been simulated using SIMION 3D software [1] to develop an electronoptic model [2]. In this work, more precise treatment of the approximation is detailed and comparison is made with corresponding experimental data. The origin of the signals features is investigated and interpreted, affording a deeper understanding into the operation and timing potential of these devices.

\section{Introduction}

The electromagnetic calorimeter of the Compact Muon Solenoid detector (CMS) [3] at the Large Hadron Collider, CERN, uses large monocrystals of the scintillator lead tungstate coupled to sensitive photodetectors, which in the endcap are vacuum phototriodes (VPT) [4] manufactured in Russia by Research Institute Electron (RIE) [5].

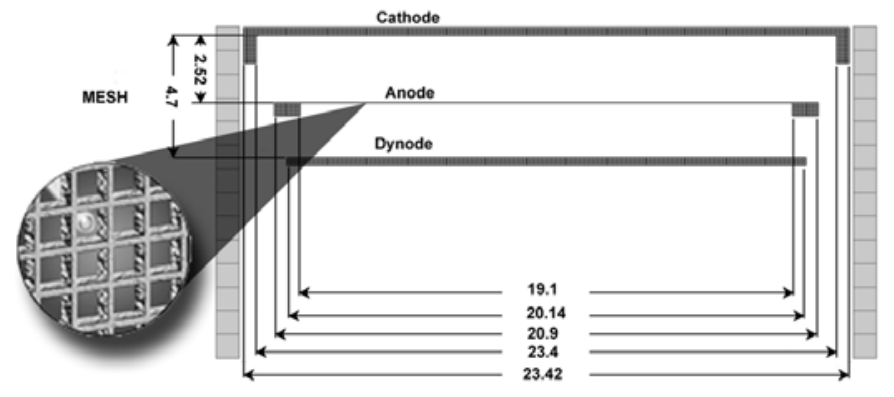

Figure 1. Illustration of VPT components (after Hobson and Yaselli [2]). The mesh anode has 50\% transparency and a pitch of 10 micrometres.

The VPT characterised in this work (see Figure 1) was supplied by RIE and is a single gain-stage photomultiplier with a diameter of $26 \mathrm{~mm}$. In our experimental arrangement, the centre of the photocathode is illuminated by a 60ps pulse from a PicoQuant SEPIA 800 diode laser $(\lambda=435 \mathrm{~nm})$. The

\footnotetext{
* Corresponding author: Tel. +44 1895 266799; Fax. +44 1895 272391; email. peter.hobson@brunel.ac.uk
} 
relatively simple planar geometry and small inter-electrode spacing suggests that the VPT should be very fast, with good timing resolution.

\section{Initial Computer Simulation: SIMION $3 D$}

The amplitude of the induced current in the anode load resistor is proportional to the intensity of the light and the gain of the VPT, controlled by the bias of the terminals: the photocathode, anode and dynode. SIMION 3D [1] has been used in conjunction with a second program written by us to simulate the behaviour of the incident photoelectrons and the resulting secondary particles in the following manner: photoelectrons from the cathode are accelerated towards the anode mesh (50\% transmission) by a high electric field. Those primary electrons which are transmitted are decelerated before being incident on the solid dynode. The dynode is manufactured from a material with a high secondary electron emission coefficient. We model the secondary electron emission from this electrode with a Poisson distribution having a mean value of 20 for an incident electron energy of $1 \mathrm{keV}$. These secondary electrons are accelerated back towards the anode, where a fraction (75\%) is collected. The remainder travel back to the dynode and are absorbed upon impact. In the event that these electrons have enough energy to produce tertiary electrons, the simulation is continued.

Using the SIMION 3D simulation, Hobson and Yaselli [2] demonstrated the reproducibility of some of the major features of the VPT response, namely the absolute gain in zero magnetic field and relative time shift. By applying Ramo's Theorem [6] the measured signal may be approximated by calculating the current, $I$, induced due to an electron moving to an electrode, i.e.:

$$
I=q \mathbf{v} \cdot \mathbf{F}_{\mathbf{k}},
$$

where $\mathbf{V}$ is the instantaneous velocity of the charge, $q$, and $\mathbf{F}_{\mathbf{k}}$ is the field that would exist due to $q$ at a distance, $d$ from the induced electrode. The solid curve in Figure 2 shows an example of the output of this model i.e. the induced current on the anode, for an anode potential $\left(V_{A}\right)$ of $1000 \mathrm{~V}$, the cathode potential $\left(V_{K}\right)$ at ground and the dynode $\left(V_{D}\right)$ at $800 \mathrm{~V}$, whilst the dashed line gives the signal following compensation for attenuation and multiplication factors which arise due to various components of the VPT. It may be seen that the zero-crossing time of the first bi-polar signal is in good agreement with the previously simulated arrival times of electrons at the anode (250ps) [2]. At later times, the signal generated by the secondary electrons emitted from the dynode due to: their direct absorption by the anode; their traversing the anode before being returned by the cathode; and their traversing the anode, returning through the mesh before being detected at the dynode. In order to best simulate the behaviour of the VPT in the experiment, the number of primary electrons has 
been calculated to be $2.6 \times 10^{6}$. The resulting signal exhibits a similar shape, although of an increased magnitude.

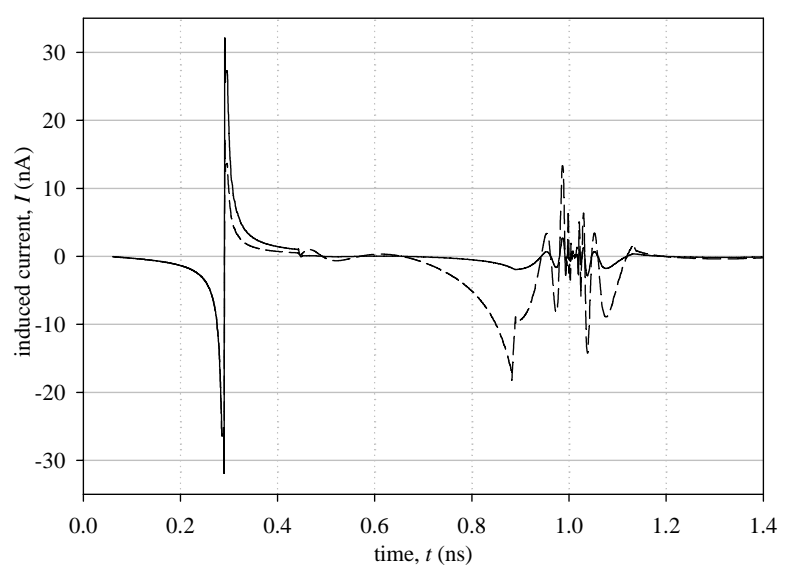

Figure 2: Simulation of current induced at anode by a single incident photoelectron: $V_{K}=0 \mathrm{~V}$, $V_{A}=1000 \mathrm{~V}, V_{D}=800 \mathrm{~V}$.

\section{Experimental Data}

Due to experimental constraints, which require the anode of the VPT to be DC coupled to the $50 \Omega$ input of the digital oscilloscope (LeCroy WaveMaster 8300A, 3GHz, 10 Gsamples/second), the potentials used in the above simulation equate to experimental values of $V_{K X}=-1000 \mathrm{~V}, V_{A X}=0 \mathrm{~V}$ and $V_{D X}=-200 \mathrm{~V}$.

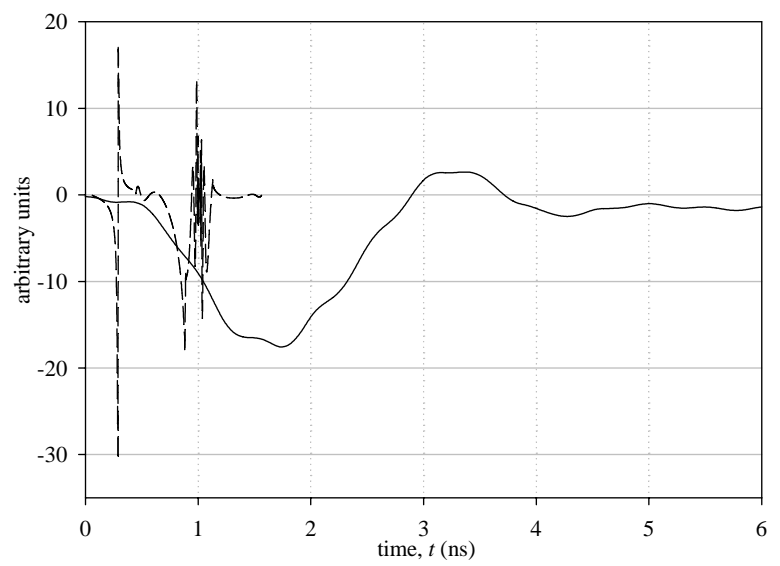

Figure 3. Comparison of simulation (---) and experimental data ( - (average of 2000 sequential traces)): the time scale of the experimental data has been shifted by $2.84 \mathrm{~ns}$ and the magnitude of the first negative peak, scaled to that seen in the simulation. 
By shifting the time-zero of the experimental data by 2.84ns, and scaling the magnitude of the negative peak, these data may be compared with the initial simulation on the same axes (see Figure 3).

The results indicate that the initial signal, seen from the SIMION simulation, is reproduced, albeit with an apparent integration due to the finite bandwidth of the experimental setup, which is dominated by the effects of the stray impedances of the VPT. In order to approximate the behaviour of the experimental apparatus upon the signal, the SIMION output has been further processed using SPICE, as discussed below.

\section{Further Simulation: SPICE}

Our simulated current data, shown in Figure 3, has been used as input to SPICE (LTSpice v.2.21) [7], such that the affect of the experimental apparatus upon the signal may be determined. A piece-wise linear approximation was made to the induced current simulated by SIMION, which was then applied to the circuit shown in Figure 4 (inset) to reflect the experimental set-up.

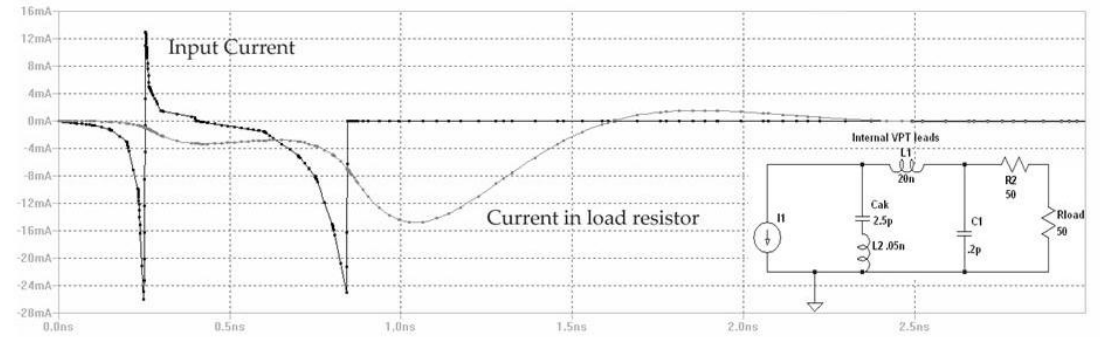

Figure 4. Comparison of input and output current in the $50 \Omega$ load resistor current from LT-Spice. The time origin of the load resistor current has been shifted to remove the effect of the delay line. Dots on the curves show the data points. Inset illustrates the circuit employed: The load resistor represents the connection to the digital oscilloscope; Cak represents the anode-cathode capacitance, and the value of $L 1$ is estimated from geometry of the internal connecting leads of the VPT; C1 represents the stray capacitance between the VPT anode socket and ground.

The resulting signal is shown in Figure 4, from which it can be seen that a plateau appears in the signal at around $0.4 \mathrm{~ns}$ after time-zero. This feature is also seen to appear between $\sim 0.1-0.5 \mathrm{~ns}$ in the experimental data (Figure 3).

Experiments have also been conducted to investigate the effect of variable $V_{A}$ and $V_{D}$ on the gain of the VPT. Maintaining constant potentials of $V_{K}=0 \mathrm{~V}$ and $V_{A}=1000 \mathrm{~V}$ whilst varying the $V_{D}$ incrementally from 0 to $800 \mathrm{~V}$ shows that the gain of the VPT increases to a maximum at $V_{D}=600 \mathrm{~V}$, beyond which the 
gain decreases, thereby confirming that the voltage gain is dependent on both the number of electrons and their velocity. By keeping the potential difference between the anode and dynode constant at $200 \mathrm{~V}$, it is evident that the voltage gain increases with increasing $V_{A}$. These general trends have also been confirmed from further computer simulation results.

\section{Conclusions and Future Work}

This paper has reported advances in the simulation process of the VPT using SIMION 3D. An experimental setup has been implemented in order to compare the simulated VPT with a production CMS VPT. Figure 4 demonstrated that the relationship between the simulated and the experimental data is that of integration due to the bandwidth limitation of the physical VPT. In order to show that the experimental timing performance of this device can match the simulated prediction, an impedance matched stripline to the anode should be built into the vacuum tube itself. With these simulation tools we are in a position to further optimise the geometric structure of vacuum photodetectors to provide very fast timing pulses for other applications.

\section{Acknowledgments}

This work is supported by the Science and Technology Facilities Council, UK.

\section{References}

[1] SIMION 3D v7.0 Scientific Instrument Services Inc. 1027 Old York Road, Ringoes, NJ 08551, USA

[2] Hobson, P R and Yaselli, I; Nuclear Instruments and Methods in Physics Research A 567 (2006) p226-229

[3] The Compact Muon Solenoid Technical Proposal, CERN/LHCC 94-38 (1994)

[4] Bell, K W et al; Nuclear Instruments and Methods in Physics Research A 469 (2001) p29-46

[5] National Research Institute Electron, Morisa Toreza Ave., 68, 194223 St. Petersburg, Russia

[6] Ramo, S; Proceedings of the IRE 27 (1939) p584-5

[7] LTSPICE, Linear Technology, 1630 McCarthy Blvd., Milpitas, CA 950357417, USA [Available: http://www.linear.com/designtools/software/] 